\title{
Laboratory Evaluation of the Validity of the Current HIV Testing Algorithm in Kenya
}

\author{
James Kimotho, ${ }^{1,}$, Zipporah Ng'ang'a ${ }^{2}$, Edna Nyairo ${ }^{1}$, Missiani Ochwoto ${ }^{1}$, Nicholas Nzioka ${ }^{1}$, \\ Francis Ogolla ${ }^{1}$, Michael Kiptoo ${ }^{1}$ \\ ${ }^{1}$ Kenya Medical Research Institute, Nairobi, Kenya \\ ${ }^{2}$ Jomo Kenyatta University of Agriculture and Technology, Institute of Tropical Medicine, Thika, Kenya
}

\section{Email address:}

jkimotho@kemri.org (J. Kimotho)

\section{To cite this article:}

James Kimotho, Zipporah Ng'ang'a, Edna Nyairo, Missiani Ochwoto, Nicholas Nzioka, Francis Ogolla, Michael Kiptoo. Laboratory Evaluation of the Validity of the Current HIV Testing Algorithm in Kenya. American Journal of Internal Medicine.

Vol. 3, No. 1, 2015, pp. 23-27. doi: 10.11648/j.ajim.20150301.14

\begin{abstract}
Background: The HIV Lateral Flow Tests (LFTs) provide a good compromise between accuracy, cost, speed and overall effectiveness. Objective: This study assessed the laboratory performance of the LFTs in the current National HIV Testing Algorithm in Kenya. Methods: Four hundred blood samples, 145 HIV positives and 255 HIV negatives, were collected from the Regional Blood Transfusion Centers in Kenya. They were analyzed using five LFTs, three of which were in the HIV Testing Algorithm in Kenya. Samples were also tested using Vironostika ${ }^{\mathrm{TM}}$ Uni-Form II Ag/Ab ELISA as a Gold Standard. The decision on the HIV status of the samples was determined by consensus status of the five LFTs. Diagnostic sensitivity (D-SN), specificity (D-SP), Positive Predictive Value (PPV) and Negative Predictive Value (NPV) were then computed together with relative Analytical sensitivity each LFT. Results: The three LFTs in the HIV Testing Algorithm in Kenya (KHB Colloidal Gold, First Response ${ }^{\mathrm{TM}} 1-2.0$ and Uni-Gold ${ }^{\mathrm{TM}} \mathrm{HIV}$ test) showed a D-SN of 100\% (95\% CI: 97.4-100.0), 96.4\% (95\% CI: 91.8 - 98.8) and 100\% (95\% CI: 97.4-100.0) respectively in relation to the Consensus status with LFTs. However, Determine ${ }^{\mathrm{TM}}$ HIV-1/2 showed the highest Analytical sensitivity when compared with two other kits in HIV Testing Algorithm in Kenya and Aware ${ }^{\mathrm{TM}}$ HIV-1/2 BSP kit. Conclusion: Though the LFTs in the current HIV Testing Algorithm in Kenya show high performance profiles, Determine ${ }^{\mathrm{TM}} \mathrm{HIV}-1 / 2$ showed higher Analytical sensitivity profile than the two HIV Screening and confirmation test kits. There is a need of reconsidering the financial savings (of 10-16\%) vis-â-vis the possibilities of missing HIV positive cases in the current HIV Testing Algorithm in Kenya.
\end{abstract}

Keywords: Lateral Flow Tests, HIV, Testing Algorithm in Kenya, Sensitivity, Predictive Value, Specificity

\section{Introduction}

The LFTs for Human Immunodeficiency Virus (HIV) provide a good compromise between accuracy, cost, speed and overall effectiveness for Point-of-Care (PoC) use in Resource-Limited Settings (RLS) [1-3]. Currently the World Health Organization (WHO) advises WHO Member States on the laboratory diagnosis of HIV including providing objective assessments of commercially available assays for detecting antibodies to HIV-1 and HIV-2 through the WHO Prequalification of Diagnostics programme. This programme provides robust assessment based on regulatory principles that consider the performance, quality and safety of the assays[4]. In Kenya, the WHO Prequalified HIV test kits are evaluated by the National HIV Reference Laboratory (NHRL) before subsequent approval by a committee of national experts appointed by the Ministry of Health. The quality of the HIV testing kits is monitored through the Proficiency Testing (PT) Programme and re-testing of a proportion of HIV samples in the NHRL by the use of Proficiency Panels where $30 \%$ HIV positive samples and $10 \%$ of HIV-negative samples are re-tested using dried blood spots[5]. While WHO uses fully characterized reference panels in the validation of HIV test kits, Kenya, like many developing countries, relies on the use of Reference kits (Gold Standard kits) in the evaluation of similar kits[6]. The use of the reference Gold Standard kits for evaluation presents some challenges when evaluating kits which are more sensitive than the Gold 
standard kit itself where wrong inference is likely to be made[7]. In February 2013, Kenya changed its National HIV Testing Algorithm into Serial Testing Algorithm (Table 1)where the HIV $(1+2)$ Antibody (KHB Colloidal Gold) (KHB Shanghai Kehua Bio-engineering Co,Shanghai, China) was adopted as a Screening Test; First Response ${ }^{\mathrm{TM}}$ 1-2.0
(PMC Medical Pty. Ltd, Daman, India) was adopted as a Confirmatory Test while Uni-Gold ${ }^{\mathrm{TM}}$ HIV test (Trinity Biotech, Oregon, USA) was adopted as a "Tie Breaker"[8]. This study was designed to further assess the validity of this Algorithm in a laboratory set-up.

Table 1. The current HIV Testing Algorithmin Kenya

\begin{tabular}{llll}
\hline KHB Colloidal Gold & First Response $^{\text {TM }}$ 1-2.0 & Uni-Gold $^{\text {TM }}$ HIV Test & HIV Status \\
\hline None Reactive & - & - & Negative \\
Reactive & Reactive & - & Positive \\
Reactive & None Reactive & Reactive & Positive \\
Reaction & None Reactive & None Reactive & Negative \\
\hline
\end{tabular}

(MoPHs Circular No. MPHS/ADM/1/12)

\section{Materials and Methods}

Four hundred blood samples, $145 \mathrm{HIV}$ positives and 255 HIV negatives, were collected from the Regional Blood Transfusion Centers (RBTCs) in Kenya (Nairobi, Nakuru, Kisumu, Embu and Mombasa) over a period of three months (May to July 2011) after removing donors' identifiers. The samples were centrifuged to produce plasma and stored at $20^{\circ} \mathrm{C}$ until use. The samples were used for development of an ELISA and a LFT for HIV guided by the prevailing consensus sequence of HIV env gene in Kenya. One of the key steps of this process was to characterize panels from the collected blood for use in the evaluation of the developed HIV kits. This was done by using six HIV antibody tests: KHB Colloidal Gold (KHB Shanghai Keshaun Bioengineering Co, Shanghai, China), First Response ${ }^{\text {TM }}$ 1-2.0 (PMC Medical Pty. Ltd, Daman, India), Uni-Gold ${ }^{\text {TM }}$ HIV test (Trinity Biotech, USA); Determine ${ }^{\mathrm{TM}}$ HIV-1/2 (Abbott Diagnostic Division,Hoofddorp,The Netherlands),Aware ${ }^{\text {TM }}$ HIV-1/2 BSP (Calypte Biomedical Corporation, Oregon, USA) and Vironostika ${ }^{\mathrm{TM}}$ Uni-Form II $\mathrm{Ag} / \mathrm{Ab}$ ELISA (bioMérieux, Marcy-l'Etoile, France). The first three kits were in the National HIV Testing Algorithm in Kenya while the fourth kit was in the immediate past National HIV Testing Algorithm in Kenya as a Screening Test. The HIV status of the samples was determined by consensus of the five LFTs and Vironostika ${ }^{\mathrm{TM}}$ Uni-Form II Ag/Ab ELISA was used as a "Gold Standard"[4].Thirty (30) HIV positive samples were pooled into three equal pools which were later double serial diluted to the $16^{\text {th }}$ dilution using pooled HIV negative samples from 10 donors. Each dilution of HIV positive pool was tested using the randomly picked test strips from the five LFTs mentioned previously in this study and the detection limits for each pool were determined for each LFT. In another experiment 250HIV negative samples by antibody tests were pooled into 10 equal pools after which HIV viral RNA was extracted from each pool using the QIAamp viral RNA kit (Qiagen, Valencia, Calif.) according to the manufacturer's protocol. For each LFT the following parameters were calculated and compared: Diagnostic Sensitivity (D-SN), Specificity (D-SP), Positive Predictive Value (PPV), Negative Predictive Value (NPV) and Analytical Sensitivity.

\section{Results}

\subsection{D-SN, D-SP, PPV and NPV Using Consensus Testing from $L F T s$}

As shown on Table 2 and Figure 1 the three(3) LFTs in the current HIV Testing Algorithm in Kenya (KHB Colloidal Gold, First Response ${ }^{\mathrm{TM}} 1-2.0$ and Uni-Gold ${ }^{\mathrm{TM}}$ HIV) test showed a D-SN of $100 \%$ (95\% CI: 97.4-100.0), 96.4\% (95\% CI: 91.8 - 98.8) and 100\% (95\% CI: 97.4-100.0) respectively in relation to the Consensus status with LFTs. The other two kits that were also in use in Kenya, Determine ${ }^{\mathrm{TM}} \mathrm{HIV}-1 / 2$ and Aware ${ }^{\mathrm{TM}} \mathrm{HIV}-1 / 2$ BSP, had a D-SN of $100 \%$ (95\%CI: 97.4$100.0 \%$ ) and $99.3 \%$ (95\% CI:96.0 to $99.9 \%)$ respectively. The KHB Colloidal Gold scored 100\% (95\% CI: 97.4-100.0) in D-SN, D-SP, PPV and NPV tests. Uni-Gold ${ }^{\mathrm{TM}}$ HIV test had the lowest D-SN but it scored 100\% (95\% CI: 97.4-100.0) in D-SP and PPV tests. The performance of the First Response $^{\mathrm{TM}} 1-2.0$ was the same as that of Determine ${ }^{\mathrm{TM}} \mathrm{HIV}$ 1/2: D-SN of $100 \%$ (95\% CI: 97.4-100.0) and PPV of $96.8 \%$ (95\% CI: 83.2 - 99.5).

Table 2. Performance of various Rapid test kits in respect to Consensus status with LFTs.

\begin{tabular}{|c|c|c|c|c|}
\hline HIV Rapid Test Kit & D-SN(95\% CI) & D-SP(95\% CI) & PPV $(95 \%$ CI $)$ & NPV $(95 \%$ CI $)$ \\
\hline Determine ${ }^{\mathrm{TM}} \mathrm{HIV}-1 / 2$ & $100(97.4-100.0)$ & $96.8(83.2-99.5)$ & $99.3(96.1-99.9)$ & $100(88.3-100.0)$ \\
\hline KHB Colloidal Gold & $100(97.4-100.0)$ & $100(88.7-100.0)$ & $100(97.4-100.0)$ & $100(88.7-100.0)$ \\
\hline Uni-Gold $^{\mathrm{TM}}$ HIV test & $96.4(91.8-98.8)$ & $100.0(88.7-100.0)$ & $100.0(97.3-100.0)$ & $86.1(70.5-95.3)$ \\
\hline First Response ${ }^{\mathrm{TM}}$ & $100(97.4-100.0)$ & $96.8(83.2-99.5)$ & 99.3(96.1-99.9) & $100(88.3-100.0)$ \\
\hline Aware ${ }^{\mathrm{TM}} \mathrm{HIV}-1 / 2$ BSP & $99.3(96.0$ to 99.9$)$ & $100.0(88.7-100.0)$ & $100.0(97.3-100.0)$ & $96.9(83.7-99.5)$ \\
\hline
\end{tabular}




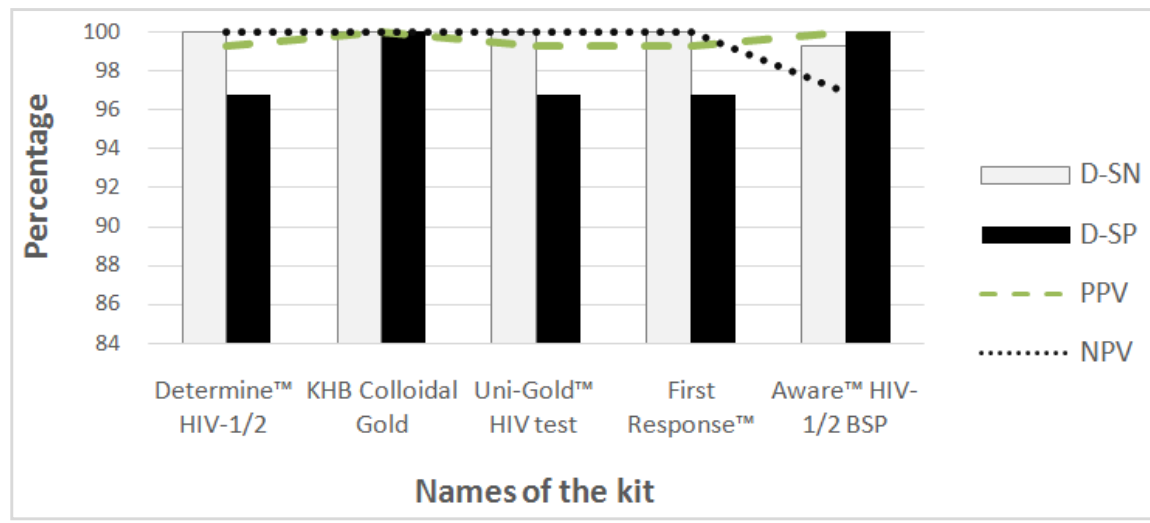

Figure 1. D-SN, D-SP, PPV and NPV in respect to Consensus status with LFTs

\subsection{D-SN, D-SP, PPV and NPV Using Consensus Testing from LFTS Using ELISA Kit}

When compared with Vironostika ${ }^{\mathrm{TM}}$ Uni-Form II Ag/Ab ELISA (Table 3 and Figure 2) as the Gold Standard the performance characteristics (D-SN, D-SP, PPV and NPV) of the LFTs were apparently reduced due higher Analytical Sensitivity of the ELISA kit. The three(3) LFTs in the current HIV Testing Algorithm in Kenya, KHB Colloidal Gold, First Response $^{\mathrm{TM}} 1-2.0$ and Uni-Gold ${ }^{\mathrm{TM}}$ HIV test, showed a D-SN of $95.9 \%$ ( 95\% CI: 91.2-98.5\%), 95.9\% (95\% CI: 91.2-97.4) and $93.8 \%$ (95\% CI: 88.5-97.1) respectively. The D-SN of Determine $^{\mathrm{TM}}$ HIV-1/2 that was the Screening kit in the immediate past HIV Testing Algorithm in Kenya HIV was 96.6\% (95\% CI: 92.2-98.9\%) which was the highest among the tested LFTs. The Aware ${ }^{\mathrm{TM}}$ HIV-1/2 BSP had a D-SN of 95.2\% (95\% CI: $90.3-98.0)$. All the LFTs tested in this study showed a D-SP of above $96.0 \%$ (95\% CI: $(79.6-99.3)$ in respect to Vironostika ${ }^{\mathrm{TM}}$ Uni-Form II Ag/Ab ELISA. All the LFTs tested displayed high PPV (above 98.6\% (95\% CI: 95.2-99.8) but low NPV, up to $73.5 \%$ (95\% CI: 55.6-87.1) as shown by Uni-Gold ${ }^{\text {TM }}$ HIV test. This implies that these LFTs had high probability of testing positive when the actual status of samples were positive but low probability of testing negative when the actual status of samples were negative.

Table 3. Performance of various Rapid test kits in respect to Vironostika ${ }^{\mathrm{TM}}$ Uni-Form II Ag/Ab ELISA.

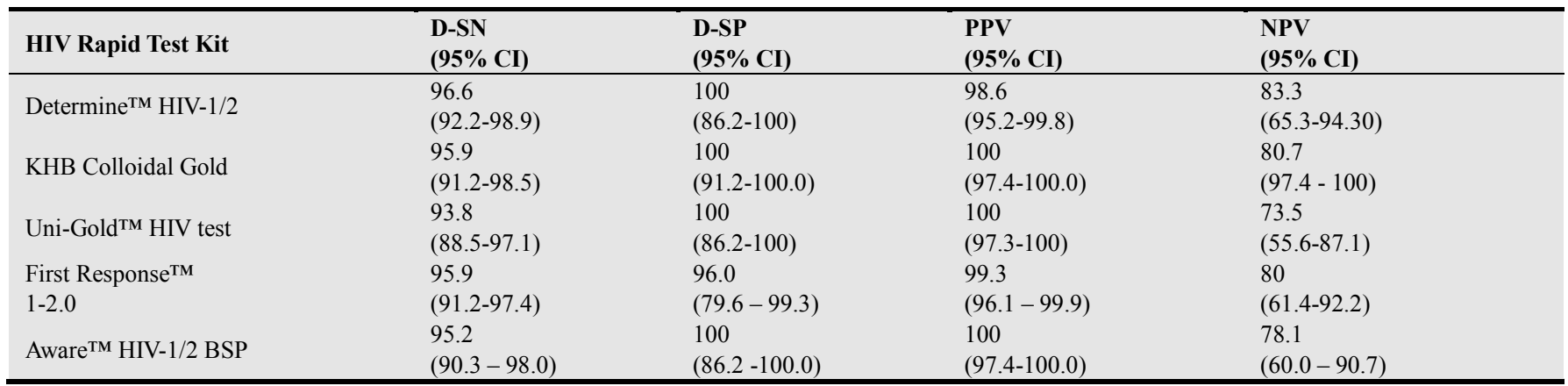

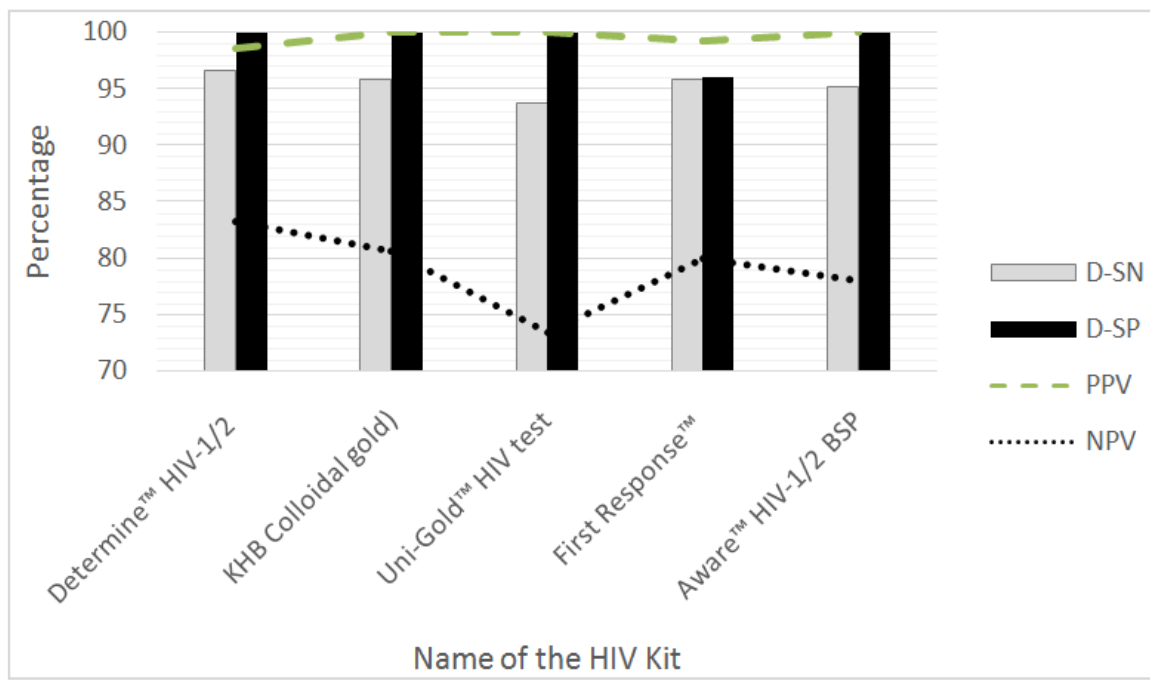

Figure 2. D-SN, D-SP, PPV and NPV in respect to Vironostika ${ }^{\mathrm{TM}}$ Uni-Form II Ag/Ab ELISA. 


\subsection{Analytical Sensitivity of the LF Ts}

In regards to testing of Analytical Sensitivity of the HIV LFTs, Determine ${ }^{\mathrm{TM}}$ HIV-1/2 showed a far much higher sensitivity in comparison with other HIV LFTs from the results of the three panels. The performance of KHB Colloidal Gold and First Response ${ }^{\mathrm{TM}}$ 1-2.0 were comparable. Uni-Gold ${ }^{\mathrm{TM}}$ HIV showed the lowest Analytical Sensitivity.

Table 4. The relative Analytical Sensitivity of HIV LFTs in Kenya using panels prepared in-house.

\begin{tabular}{|c|c|c|c|}
\hline \multirow{2}{*}{ LFTs } & \multicolumn{3}{|c|}{ Limit of detection (Double Dilution) } \\
\hline & Panel-A-15 & Panel-B-15 & Panel-C-15 \\
\hline Determine $^{\mathrm{TM}} \mathrm{HIV}-1 / 2$ & 15 & 11 & 15 \\
\hline KHB Colloidal Gold & 13 & 9 & 12 \\
\hline First Response ${ }^{\mathrm{TM}} 1-2.0$ & 11 & 11 & 12 \\
\hline Uni-Gold ${ }^{\mathrm{TM}}$ HIV Test & 9 & 5 & 8 \\
\hline
\end{tabular}

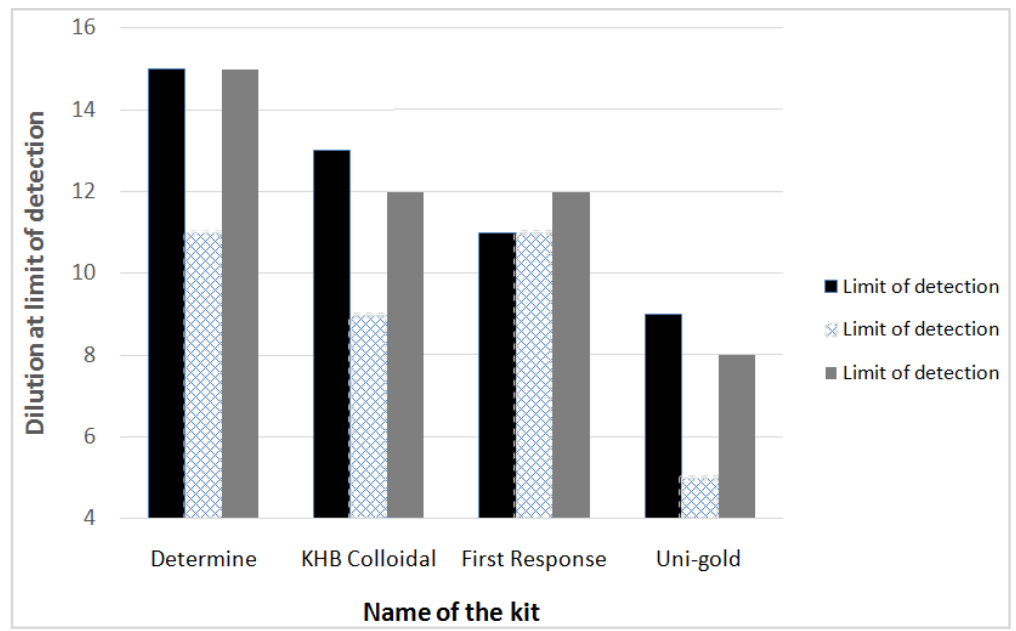

Figure 3. The relative Analytical Sensitivity of HIV LFTs in Kenya using panels prepared in-house

\subsection{RNA Extraction from HIV Negative Samples with Antibody Tests}

Out of 10 equal pools of the 250 HIV negative samples by immunological-based kits none yielded any HIV viral RNA after extraction.

\subsection{Other Observations}

With the testing of 400 samples in this study all the HIV positive samples with KHB Colloidal Goldwere also positive with the First Response ${ }^{\mathrm{TM}}$ 1-2.0 and hence there would have been no need of re-testing with Uni-Gold ${ }^{\mathrm{TM}}$ HIV test if the test were carried out using the current HIV Testing Algorithm in Kenya.

As illustrated in Table 5 the number of observed agreements between the evaluation of the LFTs by use of Consensus test from five LFTs and Vironostika ${ }^{\mathrm{TM}}$ Uni-Form II Ag/Ab ELISA was 394 (98.50\% of the observations) with Kappa $=0.967$ (95\% CI: 0.941-0.993) and P value of less than 0.0001 implying that the strength of agreement was 'very good.

Table 5. Performance of LFTs in comparison with Vironostika ${ }^{\mathrm{TM}}$ Uni-Form II Ag/Ab ELISA.

\begin{tabular}{|c|c|c|c|c|}
\hline & & Status a & 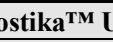 & \\
\hline & & Positive & Negative & \\
\hline Status as determined by Consensus Status & Positive & 139 & 0 & 139 \\
\hline of 6 LFTs & Negative & $\begin{array}{l}6 \\
145\end{array}$ & $\begin{array}{l}255 \\
255\end{array}$ & 261 \\
\hline
\end{tabular}

\section{Discussions}

The study observed an agreement of $98.50 \%$ (Kappa $=$ 0.967 (95\% CI: 0.941-0.993) and P value of less than 0.0001 between the evaluation of the LFTs by use of Consensus test from five LFTs and Vironostika ${ }^{\mathrm{TM}}$ Uni-Form II $\mathrm{Ag} / \mathrm{Ab}$ ELISA implying that the strength of agreement was 'very good'. These results correlate well with a number of studies that had established high sensitivity of LFTs when HIV ELISA kits were used as Gold Standard. Madisa et al. [9] found a D-SN and D-SP of $98.2 \%$ and $100 \%$ respectively for KHB Colloidal Gold against Vironostika ${ }^{\mathrm{TM}}$ Uni-Form II $\mathrm{Ag} / \mathrm{Ab}$ ELISA as Gold Standard and D-SN and D-SP of 98.2\% and $98.1 \%$ respectively for Uni-Gold ${ }^{\mathrm{TM}}$ HIV test against the same ELISA kit. Fabiani et al.[10] found a low sensitivity of the HIV Testing Algorithm using Rapid Tests of 90.6\% (95\% CI: 78.6 -96.5) when compared with an ELISA 
system.

This study did not find any sample that could require the use of a "Tie breaker' in discordant results with KHB Colloidal Gold and First Response ${ }^{\mathrm{TM}}$ 1-2.0 as prescribed in the Kenya HIV Testing Algorithm. According to the findings of the study the Uni-Gold ${ }^{\mathrm{TM}}$ HIV test had the lowest Diagnostic and Analytical sensitivities and its specificity profile was not outstanding in comparison with other LFTs. Its preference as a "tie breaker" in the Kenyan HIV Testing Algorithm is debatable given that other HIV Testing Algorithms suggest the use of "tie breaker" testing kits that are more sensitive and specific than kits that are used for either Screening or Confirmation, such as nucleic acid amplification tests (NAATs) or ELISA though the high costs of these tests tend to limit their use especially in developing countries[11].

Determine $^{\mathrm{TM}} \mathrm{HIV}-1 / 2$ showed the highest values of Analytical Sensitivity and D-SN with ELISA as Gold Standard implying that the current National HIV Testing Algorithm in Kenya could benefit more by using this kit as a Screening Test. The results could point out the fact that the change of National HIV Testing Algorithm to slightly less sensitive kits could give an inaccurate HIV prevalence in Kenya in the long run. Possibly, the only reason why Determine $^{\mathrm{TM}}$ HIV-1/2 was not included in the current National HIV Testing Algorithm in Kenya was that it was slightly more expensive than other kits that were tested, costing USD 0.87 per test (in 2013) as compared to USD 0.77 for First Response ${ }^{\mathrm{TM}}$ 1-2.0 and USD 0.79 for KHB Colloidal Gold over the same period[12]. However, the cost of the missing some HIV positive cases should not be forgotten when such a decision is to be made.

Efforts to extract HIV viral RNA from the 255 HIV negative samples by antibody tests did not yield any RNA positive sample. This could have been due to the fact that either none of these samples were from a subject with acute HIV infection that had been found in some studies to be less than $1 \%[13]$ or the sample size in this study was too small to detect any acute infection among the HIV negative samples.

The main limitations of this study include the use of plasma in testing for HIV rather than whole blood. However, previous studies have shown very close correlation between the results found with plasma and those found with whole blood[7].

In conclusion this study has established that the performance of the LFTs in the current HIV Testing Algorithm is Kenya is generally high. The study, however, recommends the review of the Algorithm to have Determine $^{\mathrm{TM}} \mathrm{HIV}-1 / 2$ as the Screening Test as it is the most sensitive LFT in the Kenyan market today.

\section{Acknowledgements}

We are grateful to KEMRI-CDC for facilitating procurement of the HIV diagnostic kits which have been used in this study, Director Kenya National Blood Transfusion Services for allowing us to use rejected donors' blood for the study and staff, KEMRI Production Department for their moral support of this study and Director KEMRI for permission to publish the findings.

\section{References}

[1] WHO-EMRO, Rapid HIV Tests: Guidelines for use in HIV testing and counseling services in resource-constrained setting. 2004.

[2] Van Binsbergen, J.d.R., D.; Peels, H.; Dries, C.; Scherders, J.; Koolen, M. Zekeng, L. and Gürtler, L.G., Evaluation of a new third generation anti-HIV-1/anti-HIV-2 assay with increased sensitivity for HIV-1 group O. Journal of Virological Methods, 1996. 60(2): p. 131-7.

[3] Wong, E., Y.; Indira, K. and Hewlett, 1, HIV Diagnostics: Challenges and Opportunities: Rapid \& PoC Testing in RLS. HIV Therapy, 2010. 4(4): p. 399-412.

[4] WHO, HIV assays operational characteristics: HIV rapid diagnostic tests (detection of HIV-1/2 antibodies). report 2013. 17.

[5] National AIDS and STI Control Programme, M.o.P.H.a.S., Kenya, Guidelines for HIV Testing and Counselling in Kenya. NASCOP, 2008.

[6] Plate, D.K., Evaluation and implementation of rapid HIV tests:the experience in 11 African countries. AIDS Res Hum Retroviruses, 2007. 23: p. 1491-1498.

[7] TDR, Evaluation of diagnostic tests for infectious diseases: general principles. 2010

[8] Ministry of Public Health and Sanitation, K., Circular No. MPHS/ADM/1/12 dated 6th February 2013

[9] Madisa, M.C., S; Kgomotso, S.; Taurayi, T, Performance of Rapid HIV Testing by Lay Counsellors in the Field during the Behavioural and Biological Surveillance Survey among Female Sex Workers and Men who have Sex with Men in Botswana. Journal of acquired Immune Deficiency Syndromes

[10] Fabiani, M.N., B.; Biryahwaho, B.; Ouma, J.; Ninci, A.; Declich, S., Evaluating HIV testing algorithms for research, diagnosis and surveillance. health policy and development, 2005. 3(1).

[11] Slev, P., The changing landscape of HIV diagnostics. Medical Laboratory Observer: Cover Story, 2012.

[12] Lyer, P., Mwai, D., Ng'ang'a, A., Costing Kenya's current and proposed HIV testing and Counseling Algorithms. Health Policy Project 2013.

[13] Pebody, R., Large US study shows which HIV tests are most accurate; Antibody-only tests need to be replaced with combination tests; Antibody-only tests need to be replaced with combination tests. NAM AIDS MAP, 2014. 